\title{
Diagnóstico de la cultura organizacional actual y deseada: estudio de caso de una empresa comercializadora de acabados y materiales para la construcción
}

Diagnosis of the current and desired organizational culture: case study of a company that sells construction materials and finishes

Diagnóstico da cultura organizacional atual e desejada: estudo de caso de uma empresa que comercializa materiais de construção e acabamentos

Mtro. Igor Piotr Beraud Martínez igorberaud@hotmail.com https://orcid.org/0000-0003-1038-1022

Universidad Autónoma de Sinaloa

\section{Dr. José Gerardo Ignacio Gómez Romero} gerardoignaciog@yahoo.com.mx https://orcid.org/0000-0002-6322-6133 Universidad Juárez del Estado de Durango

\section{Dr. Rosalío Tortolero Portugal} chalioby@hotmail.com https://orcid.org/0000-0002-4526-7417 Centro Educativo de Líderes Durango

Dra. María Brenda González Herrera brenda.gonzalez@ujed.mx https://orcid.org/0000-0003-3697-2074 Universidad Juárez del Estado de Durango

\section{RESUMEN}

La cultura organizacional es de suma importancia para lograr una ventaja competitiva. Cada organización desarrolla una cultura propia, única e intransferible que la diferencia de sus competidores. El propósito de la presente investigación es diagnosticar la cultura dominante y la cultura deseada de una empresa comercializadora de acabados y materiales para la construcción de la ciudad de Mazatlán Sinaloa México. Para la recopilación de la información se utilizó la técnica de la encuesta. El instrumento de medición empleado es el que contempla el Marco de Valores en Competencia (MVC) y el Organizational Culture Assessment Instrument (OCAI), se aplicó en el mes de marzo del año 2021 a 40 trabajadores que representa el total de la planta laboral de la organización. Se concluye que la cultura dominante fue la de mercado y la cultura deseada fue la de clan.

Palabras clave: Cultura organizacional, cultura dominante y cultura deseada.

\begin{abstract}
Organizational culture is of the utmost importance to achieve a competitive advantage. Each organization develops its own, unique and non-transferable culture that differentiates it from its competitors. The purpose of this research is to diagnose the dominant culture and the desired culture of a commercialization company of finishes and materials for the construction of the city of Mazatlán Sinaloa Mexico. The survey technique was used to collect the information. The measurement instrument used is the one that contemplates the Competency Values Framework (MVC) and the Organizational Culture Assessment Instrument (OCAI), it was applied in March 2021 to 40 workers representing the total workforce of the organization. It is concluded that the dominant culture was that of the market and the desired culture was that of the clan.
\end{abstract}

Keywords: Organizational culture, dominant culture and desired culture.

\section{RESUMO}

A cultura organizacional é de extrema importância para obter uma vantagem competitiva. Cada organização desenvolve sua própria cultura única e intransferível que a diferencia de seus concorrentes. O objetivo desta pesquisa é diagnosticar a cultura dominante e a cultura desejada de uma empresa de comercialização de acabamentos e materiais para a 
construção da cidade de Mazatlán Sinaloa México. A técnica de pesquisa foi usada para coletar as informações. a organização. Conclui-se que a cultura dominante era a do mercado e a cultura desejada era a do clã.

Palavras-chave: Cultura organizacional, cultura dominante e cultura desejada.

\section{INTRODUCCIÓN}

Mantener su negocio en el transcurso del tiempo, es una de las grandes preocupaciones para cualquier empresario, además de tener una rentabilidad constante (Guizar, 2004). Lo anterior, resulta cada vez más complicado, sobre todo en un entorno de constantes cambios y amplia competencia doméstica internacional e inclusive digital. A pesar de ello, la necesidad de formular un camino adecuado por el cual transitar en la vida empresarial, ha sido motivo de interés. La falta de desarrollo de las habilidades gerenciales para liderar y dirigir a los equipos de trabajo dentro de las organizaciones, termina afectando la operación de la empresa, traduciéndose en baja rentabilidad o muy malos resultados. Al no tener los suficientes ingresos, las empresas no pueden innovar y, mucho menos, lograr una ventaja competitiva sobre sus competidores. No obstante, hay empresas cuyos ingresos son lo suficientemente grandes para invertir en capacitación, renovación de infraestructura, pero, tampoco lo hacen, porque los dueños lo consideran innecesario.

Estos factores entre algunos otros, conllevan a las empresas al fracaso. Por ello, ha existido un interés compartido en el ámbito académico y empresarial, en la lógica de encontrar formas de satisfacer dichas inquietudes. Entre estos esfuerzos, surge el tema de cultura organizacional, resultado de una línea de trabajo surgida a inicios del siglo XX, ligada a las relaciones humanas y las ciencias del comportamiento, centrada en un cambio de pensamiento del líder, una preocupación genuina por el hombre, sus interacciones y cómo éstas tienen efectos sobre la productividad organizacional (Albarrán-Carrera, 2010). De aquí surge la formulación del siguiente problema: ¿cuál es la cultura organizacional actual y deseada según la perspectiva de los trabajadores de la empresa estudiada? En este sentido, el objetivo general de la presente investigación es diagnosticar la cultura organizacional actual y preferida de una empresa comercializadora de acabados y materiales para la construcción ubicada en el estado de Sinaloa México, además, como objetivo específico identificar si existen diferencias entre las dimensiones de la cultura organizacional. Así mismo, se justifica por la relevancia que tendrá el diagnóstico para el líder de la organización, y de esta manera valore la perspectiva que tienen sus trabajadores acerca de la cultura actual pero sobre todo estimé cuales son las prácticas organizacionales, los criterios de éxito, la cohesión y el liderazgo que prefieren sus trabajadores para sentirse motivados y alcanzar los objetivos planteados.

La hipótesis general de la investigación es que la cultura organizacional actual y la deseada según las perspectivas de los trabajadores de la empresa estudiada son homogéneas, es decir no presentan diferencias.

Se realizó la fundamentación teórica sobre la variable de estudio que corresponde a la cultura organizacional para evaluar la conveniencia del empleo del Marco de Valores en Competencia y su idoneidad en el contexto local. Gran parte de las investigaciones sobre el tema han sido realizadas en contextos distintos al local, por lo cual, resulta de gran relevancia conocer si una cultura organizacional cercana a las percepciones del personal tendrá mejores resultados en las operaciones diarias, el comportamiento y conductas de los empleadores dentro de cualquier organización de la localidad, toda vez que la evidencia obtenida muestra que la cultura actual afecta el comportamiento del personal y en consecuencia el desempeño del negocio. Además, en el procedimiento metodológico se indica el tipo y diseño de investigación, como también, las técnicas e instrumento aplicados para la recolección de datos. Los resultados muestran el diagnóstico de la cultura organizacional deseada y actual de la organización y por último, las conclusiones mencionan los hallazgos más relevantes. 


\section{FUNDAMENTACIÓN TEÓRICA.}

El concepto de cultura organizacional surge a finales de la década de los setentas, inicios de los ochentas, en los trabajos de Pettigrew (1979), quien describió a la cultura organizacional como el sistema de significados aceptados pública y colectivamente que operan para un grupo dado en un momento determinado. Este sistema de términos, formas, categorías e imágenes interpreta la propia situación de un pueblo para sí mismo (Pettigrew 1979). Por su parte, Ouchi (1982), presenta los resultados de un estudio comparativo de culturas entre empresas estadounidenses ubicadas en Japón y empresas niponas llegadas a la tierra de la libertad.

Así mismo, es posible identificar al menos, un par de vertientes más acerca de la cultura organizacional, por un lado, aquellos trabajos realizados desde disciplinas como la sociología, la antropología, entre otras y, por el otro, la del management donde están directamente relacionadas la administración y los estudios organizacionales, siendo la segunda de interés para esta investigación (Albarrán-Carrera 2010). Dentro de esta línea administrativa-organizacional también existe una división en la forma de abordar el tema: desde una perspectiva académica y la encaminada a la consultoría.

No obstante, como señala Pacheco-Espejel (2015), la consultoría adolece de un respaldo teórico específico del fenómeno organizacional, es decir, está más centrada en el diseño y aplicación de soluciones puntuales a problemas concretos localizados en cualquiera de las áreas funcionales de la organización, lo cual, es traducible en un aporte casi nulo al estudio de dicho campo. En este orden de ideas, los estudios organizacionales y administrativos permiten hipotetizar $\mathrm{y}$ contrastar los planteamientos formulados, valorar $\mathrm{y}$ analizar resultados, su viabilidad $\mathrm{y}$ confiabilidad, servir como punto de referencia para investigaciones similares o abrir la posibilidad a nuevos planteamientos, en cualquiera de los casos, enriquecer el conocimiento existente.

Adicionalmente, la cultura organizacional hace referencia a los eventos observados dentro de cualquier organización. Una organización no necesariamente es una empresa. Según Hodge, Anthony y Gales (2003), una "organización se define como dos o más personas que trabajan en colaboración y en conjunto dentro de unos límites identificables para alcanzar una meta u objetivo en común". Dichos fenómenos están relacionados con los patrones de comportamiento, valores y creencias compartidos por los integrantes de una agrupación (Andrade, 2011). Es decir, el aspecto cultural puede llegar a ser un factor determinante de éxito o fracaso, en muchos sentidos, para cualquier empresa u organización (Cameron y Quinn, 2011).

A su vez, el ámbito de estudio no queda limitado a este aspecto, sino, también abarca fenómenos relacionados con intercambios culturales y los efectos sobre el desempeño organizacional. Un claro ejemplo de ello es lo trabajado por Ouchi (1982) y su teoría Z, la cual, hace referencia a los resultados presentados por empresas norteamericanas al instalarse en Japón, el tipo de estrategia utilizado para entrar al mercado nipón y las diferencias culturales experimentadas por los empleados al estar en un contexto distinto. De forma similar, recopila los casos de empresas japonesas tratando suerte al instalarse en territorio estadounidense, conservando en ambos casos la toma de decisiones ejecutiva en personal de sus respectivos países de origen.

$\mathrm{Al}$ respecto, un elemento importante de análisis dentro de los estudios culturales está ligado a los valores compartidos por el grupo estudiado, en este caso, la confianza jugó un rol importante en lo observado por Ouchi (1982) al desarrollar la teoría Z. Lo anterior, tiene una relación muy estrecha con las diferencias culturales existentes entre estadounidenses y japoneses. Para los norteamericanos resultaba muy atractivo el tener una carrera con logros y crecimiento acelerado, por su parte, los nipones entienden de forma distinta el manejo organizacional, conciben la paciencia y la confianza como valores primordiales en el crecimiento de su carrera laboral. 
Teniendo como hilo conductor, lo mencionado anteriormente, Geert Hofstede, es otro de los académicos quien ha trabajado los impactos interculturales en los rendimientos organizacionales.

Uno de sus planteamientos giraba en torno a las confrontaciones culturales, es decir, cómo la cultura de un país influye en la forma de pensar y actuar de los individuos trasladando estos comportamientos a las organizaciones, generando incompatibilidad entre la cultura de una empresa y la cultura general, aun, cuando la cultura organizacional puede ser gestionada o cambiada, según lo citado por Tarapuez-Chamorro (2016).

Otro importante autor cuyo trabajo retoma los planteamientos vertidos con anterioridad es Edgar H. Schein, aunque, sus principales aportes están encaminados en una línea distinta; definir la cultura organizacional y sentar las bases para su análisis desde una perspectiva cualitativa, por ello, resulta primordial para el autor realizar observaciones in situ, pues una parte importante del estudio de la cultura dentro de cualquier organización es la observación, principalmente, poner atención en los pequeños detalles sobre la forma como realiza el personal sus actividades diarias, si existen rangos jerárquicos establecido y marcados; cómo se saludan entre compañeros de igual nivel, subordinados y superiores, entre otros varios aspectos de importancia, según lo descrito por Schein (2010). Así mismo, Schein (2010) con base en la experiencia de sus diferentes intervenciones organizacionales, sugiere que la cultura de cualquier organización está dividida en tres niveles: 1) artefactos, 2) creencias y valores propuestos y 3) supuestos básicos subyacentes, los cuales, son descritos por Minsal-Pérez y Pérez-Rodríguez (2007) de la siguiente manera:

- Nivel 1. Es el más visible e incluye el espacio físico, capacidad tecnológica, lenguaje, conducta observada en los miembros de una organización, producciones artísticas; en general, se compone de todos aquellos elementos que pueden captarse con nuestros sentidos.

- Nivel 2. Los que la organización y sus miembros piensan que deben ser, en función de lo cual actúan de una u otra manera.

- Nivel 3. Formado por una serie de presunciones básicas, invisibles y preconscientes que se dan por sentadas, son cuestiones indiscutibles y asimiladas por el personal, que piensa que determinadas cosas son así porque no pueden ser de otro modo.

Los puntos anteriores estarían asociados a la llamada gestión de la cultura organizacional.

Cuando se dice que la cultura es dinámica, hace referencia a aquella parte, la cual, puede ser cambiada y adaptada a las nuevas necesidades del entorno social y organizacional. Entonces, al gestionar la cultura organizacional y tratar de cambiarla, se estaría hablando de los dos niveles primarios. No obstante, existen detractores de este planteamiento, quienes afirman lo difícil o casi imposible de lograr el cambio cultural en las organizaciones. Empero, lo anterior podría ser explicado de la siguiente manera, el tercer nivel está relacionado con la parte informal, los aspectos intangibles, con la hegemonía de "lo que es" sobre "lo que debiera ser", la prevalencia de comportamientos y conductas dadas como válidos $\mathrm{y}$, en consecuencia, aceptados como tal, esto es lo difícil de cambiar.

\subsection{Diagnóstico de la Cultura Organizacional}

Una forma de iniciar el cambio cultural es a través del diagnóstico de la cultura actual de una organización. Para ello es necesario informar al personal sobre la intención de cambio, así mismo, esté enterado de la existencia de una cultura; cuáles son sus elementos distintivos y constitutivos, cómo afecta a sus actividades laborales, sus comportamientos y el desempeño de la institución. En este orden de ideas, una manera de hacerlo es a través del empleo del Competing Values Framework (MVC) o Modelo de Valores en Competencia (MVC) desarrollado por Cameron

y Quinn (2011). No es la única metodología existente, sin embargo, al ser una herramienta ampliamente utilizada en diferentes contextos, sectores, ámbitos y estudios (Chuc, 2017, Chuc,2018; Ibarra-Michel, et al., 2017; Villarreal,et al., 2012,) a nivel mundial, incluidos los latinoamericanos, ofrece confianza en su aplicabilidad. Así mismo, Cameron y Quinn (2011), 
consideran a la cultura como un elemento de suma importancia, porque a partir de ella son definidos los valores centrales, supuestos, interpretaciones y enfoques característicos y diferenciadores de una organización.

Retomando el citado modelo, éste consta de dos dimensiones (Chuc, 2018), las cuales, al ser expresadas de manera lineal cada una de las rectas en sus respectivas puntas o extremos representan una serie de valores completamente opuestos, de allí su nombre de Modelo de Valores en Competencia, como puede observarse en la Figura 1. Por un lado, una de estas dimensiones representa en una extremidad una disposición organizacional hacia el control, la estabilidad y el orden. Así, el lado contrario muestra una tendencia hacia el cambio, la flexibilidad y la capacidad de adaptación. Por su parte, la segunda dimensión hace alusión a su orientación, ya sea interna (integración) o externa (diferenciación y rivalidad), según lo señalado por Florido y Zesaty (2012).

Al realizar una representación gráfica consistente en un cruce de las líneas antedichas, el resultado obtenido es un plano cartesiano donde el eje vertical o de las ordenadas (Y) en su parte superior está asociado a; flexibilidad, quedando control en el extremo inferior.

Para Chuc (2018), se trata de una relación encontrada entre estabilidad y flexibilidad, es decir, "hace referencia a si la organización considera más importante el orden y el control (estabilidad) o el dinamismo y la discrecionalidad (flexibilidad)".

De esta forma, el eje de las abscisas u horizontal (X) quedaría conformado por los conceptos; interno (izquierda) y externo (derecha) ubicados a los extremos de la línea en sentido encontrado. La contraposición de estos valores refiere al hecho de si existe una mayor preocupación por parte de los líderes en atender las necesidades internas de la organización o, por el contrario, prestar más atención a las demandas del contexto exógeno.

\section{Figura 1}

Modelo de Valores en Competencia (MVC)

Flexibilidad, cambio, capacidad de adaptación

$(\mathbf{Y})$

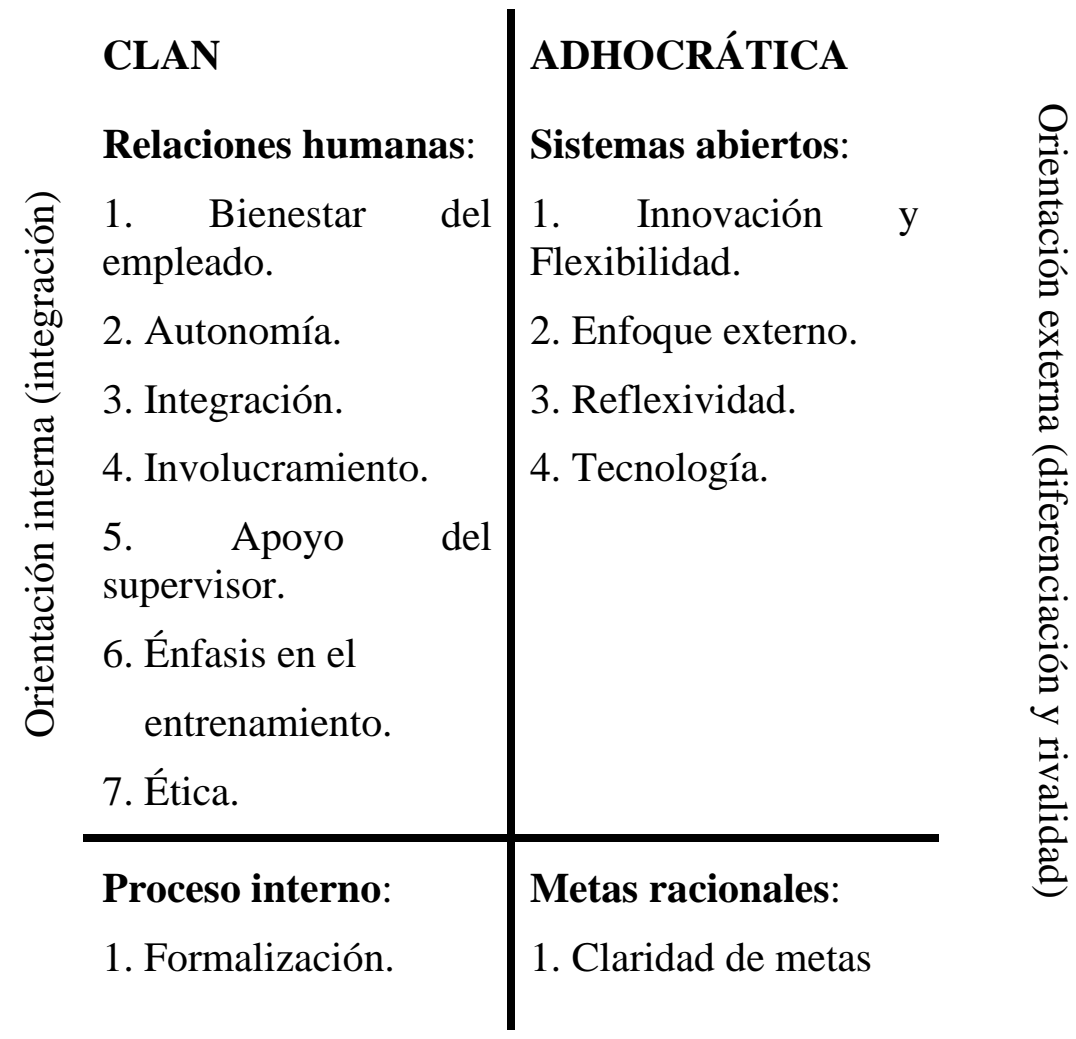




\begin{tabular}{l|l}
\hline 2. Tradición. & 2. Esfuerzo. \\
3. Centralización. & 3. Eficiencia. \\
4. Complejidad. & 4. Calidad. \\
5. Tensión y estrés. & 5. Presión para \\
6. Control. & $\begin{array}{l}\text { producir. } \\
\text { 7. Poder. }\end{array}$ \\
8. Gobernabilidad. & del desempeñontación \\
JERÁRQUICA & MERCADO
\end{tabular}

Control, estabilidad, orden

Fuente: Adaptado de Cameron y Quinn (2011), Hernández y Valencia (2012).

Como puede observarse en la Figura 1, ésta contiene los nombres de los cuatro tipos de cultura organizacional propuestos por Cameron y Quinn (2011) cada una ubicada en un cuadrante distinto e independiente, pudiéndose observar cómo resultan ser opuestas una de la otra. A su vez, Salas-Arbeláez et al., (2017) realizan una interpretación de las características de dichas tipologías:

La cultura jerárquica; se caracteriza por un ambiente formalizado, apto y con una estructura adecuada para ejercer la labor que se disponga; los procesos estandarizados definen lo que los individuos realizan en la organización.

La cultura de mercado; está principalmente orientada hacia el entorno externo en vez del interno, se centra en transacciones con diferentes grupos externos con los cuales se involucra la organización, como lo pueden ser proveedores, clientes, contratistas, etc. Este opera en el ámbito competitivo y el intercambio monetario.

La cultura clan; es llamada de esta forma debido a su gran parecido al tipo de organización de un grupo familiar. Se caracteriza por la gran cohesión entre sus miembros, la participación, descritas como «una familia extensa» tanto que no parecían entidades económicas. En este tipo de cultura, en vez de las reglas como la jerárquica y de la competencia como la de mercado, prevalecen valores como el trabajo en equipo, programas que hacen mucho más activa la participación de los empleados y el compromiso de la compañía para con sus miembros.

La cultura adhocrática; tiene como principal característica y suposición que la innovación y las iniciativas pioneras conducen las organizaciones al éxito; su principal objetivo es desarrollar nuevos productos y servicios y estar preparados para lo venidero, por lo tanto, los altos mandos se enfocan en generar emprendimiento y creatividad.

\section{PROCEDIMENTOS METODOLÓGICOS}

La investigación es de tipo no experimental, en cuanto a su temporalidad es transversal, ya que se desarrolla en un momento específico y no se toman mediciones o captan resultados a lo largo del tiempo, es descriptiva porque muestra el análisis de la percepción acerca de la variable de estudio que corresponde a la cultura organizacional. 
Para la recopilación de la información se utilizó la técnica de la encuesta. El instrumento de medición empleado es el que contempla el Marco de Valores en Competencia (MVC) y el Organizational Culture Assessment Instrument (OCAI) diseñado por Cameron y Quinn (2011), el cual tiene como objetivo identificar el tipo de cultura que predomina en la actualidad, además muestra el tipo de cultura deseada por parte de los trabajadores. Se aplicó en el mes de marzo del año 2021 a 40 trabajadores y directivos que representan el total de la planta laboral de la organización, distribuidos en una matriz ubicada en la ciudad de Mazatlán, Sinaloa, y en 3 sucursales, dos de ellas en el mismo estado, ubicadas en la ciudad de Culiacán y los Mochis y la tercera en la ciudad de Guadalajara Jalisco México, donde el $27.5 \%$ de la muestra son mujeres y el $72.5 \%$ hombres.

El OCAI, es un cuestionario de escala ipsativa diseñado para diagnosticar o detectar cuál es el tipo de cultura hegemónica en una organización. Así mismo, dicho instrumento permite conocer la tipología deseada por los integrantes de la agrupación. Esto es posible porque identifica y analiza seis categorías o dimensiones nodales que corresponden a; 1. Características dominantes; 2. Liderazgo organizacional; 3. Gestión de los empleados; 4. Cohesión organizacional; 5. Énfasis estratégico y 6. Criterios de éxito (Gómez-Romero, 2008).

Estas seis dimensiones permiten entender como es operada "en el ahora" la organización, pero, también es posible conocer cuáles serían los cambios o mejoras esperados por los integrantes en cada una de estas dimensiones, dando como resultado seis bloques con cuatro ítems cada uno, siendo en total veinticuatro, marcados por las primeras cuatro letras del abecedario correspondiendo a las tipologías culturales $\mathrm{A}=\mathrm{Clan}, \mathrm{B}=$ adhocrática, $\mathrm{C}=$ jerárquica, $\mathrm{D}=$ mercado. Así mismo, contiene dos columnas marcadas como actual y preferida, donde, el participante debe distribuir cien puntos entre los cuatro ítems correspondientes a un bloque o dimensión, asignando la puntuación más alta a aquellos que más se acerque a lo que vive en su empresa buscando conocer la cultura dominante en la actualidad (González, Liquidano y Carlos-Ornelas, 2012). Respecto a la cultura deseada, la dinámica es similar, enfocándose solamente en la columna marcada como preferida, distribuyendo los 100 puntos en los cuatro ítems de cada dimensión, asignando mayor calificación donde es deseada la presencia de un cambio. Una vez contestado el instrumento, son sumados todos los ítems marcados como A, B, C y D y finalmente son promediados.

\section{RESULTADOS Y DISCUSIÓN}

El procesamiento de los datos después de la aplicación del OCAI, muestran como resultado el diagnóstico de la cultura organizacional de la empresa estudiada. En este sentido, la figura 2, muestra el comparativo gráfico entre la cultura dominante y la preferida. Se puede observar, que existe una orientación hacia la tipología de mercado como la cultura actual y la tipología clan como la cultura deseada. Haciendo referencia a la figura 1 denominada Modelo de Valores en Competencia (MVC), el análisis muestra que la mayoría de los procesos y actividades realizadas de forma cotidiana, están enfocados en la obtención de resultados económicos adecuados, teniendo las ventas como un factor clave.

Figura 2.

Comparativo gráfico de la Cultura Actual y Preferida. 


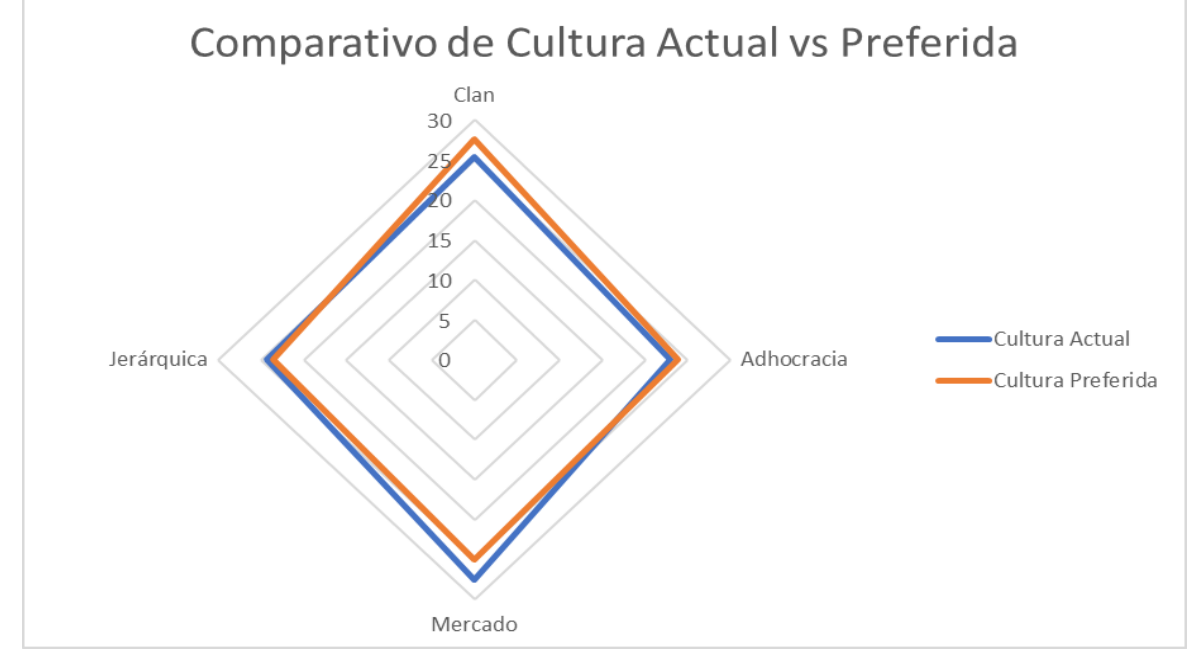

Fuente: Elaboración propia.

Así mismo, la tabla 1, muestra los porcentajes correspondientes a cada una de las tipologías, tanto de la cultura actual como la deseada, donde se puede observar los valores equilibrados, confirmando la coexistencia de los cuatro tipos de cultura explicados por Cameron y Quinn (2011), además se observa un aparente balance en la convivencia de dichas tipologías, sin embargo, la tipología de Clan como cultura preferida presenta el mayor porcentaje con $27.6 \%$.

\section{Tabla 1.}

Comparativo por porcentajes de Cultura Actual y Preferida.

\begin{tabular}{lrrrc}
\hline \multicolumn{1}{c}{ Cultura } & Clan & Adhocracia & Mercado & Jerárquica \\
\hline Actual & 25.3 & 22.9 & $\mathbf{2 7 . 5}$ & 24.3 \\
Preferida & $\mathbf{2 7 . 6}$ & 23.9 & 25 & 23.6 \\
\hline
\end{tabular}

Fuente: Elaboración propia

En relación, a las características de la cultura dominante, la figura 3 muestra la hegemonía de la cultura de Mercado, así mismo, se puede observar como en la cultura preferida existe un aumento en el porcentaje que corresponde a la adhocracia, coincidiendo con las percepciones de los trabajadores en relación a que son escuchados y considerados en la mejora e innovación de los procesos correspondientes a sus áreas de trabajo.

Figura 3.

Comparativo gráfico de la dimensión: Características Dominantes.

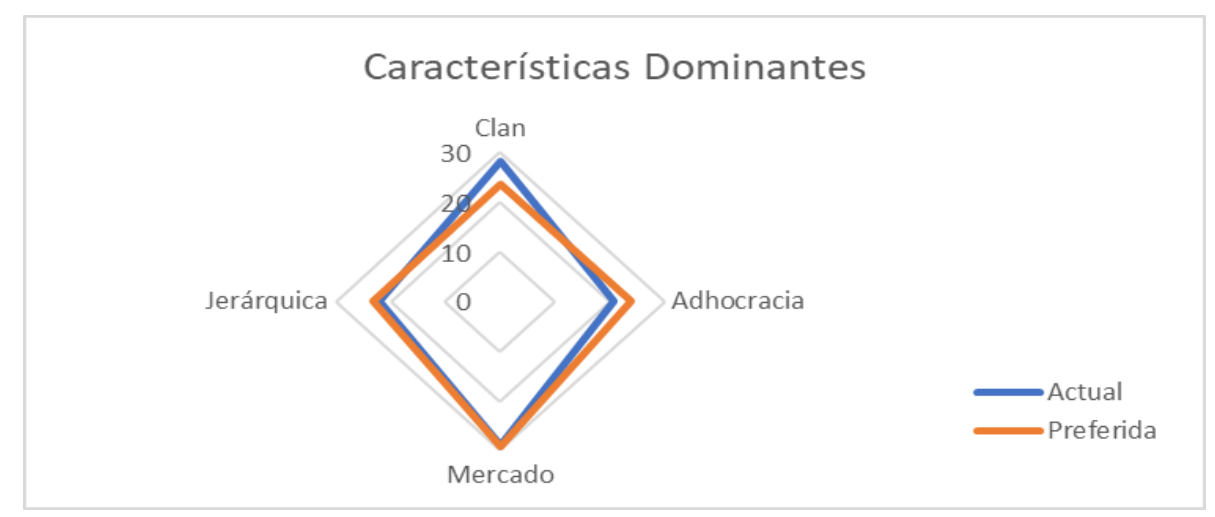


Fuente: Elaboración propia.

En este sentido, la tabla 2, muestra la normalización de las otras tipologías de la cultura deseada, teniendo un promedio de $23 \%$. En lo que corresponde a la tipología de Clan se puede observar que tuvo un descenso del $5 \%$.

Tabla 2.

Comparativo por porcentajes, dimensión: Características Dominantes

\begin{tabular}{|c|c|c|c|c|}
\hline Cultura & Clan & Adhocracia & Mercado & Jerárquica \\
\hline Actual & 28.2 & 20.8 & 29 & 22.1 \\
\hline Preferida & 23.7 & 23.9 & 29.3 & 23.1 \\
\hline
\end{tabular}

Fuente: Elaboración propia

La figura 4 muestra el análisis realizado en la dimensión de liderazgo organizacional, donde se puede observar una inclinación a la cultura de tipo Clan, tanto en la actual como en la preferida.

Figura 4.

Comparativo gráfico de la dimensión: Liderazgo Organizacional.

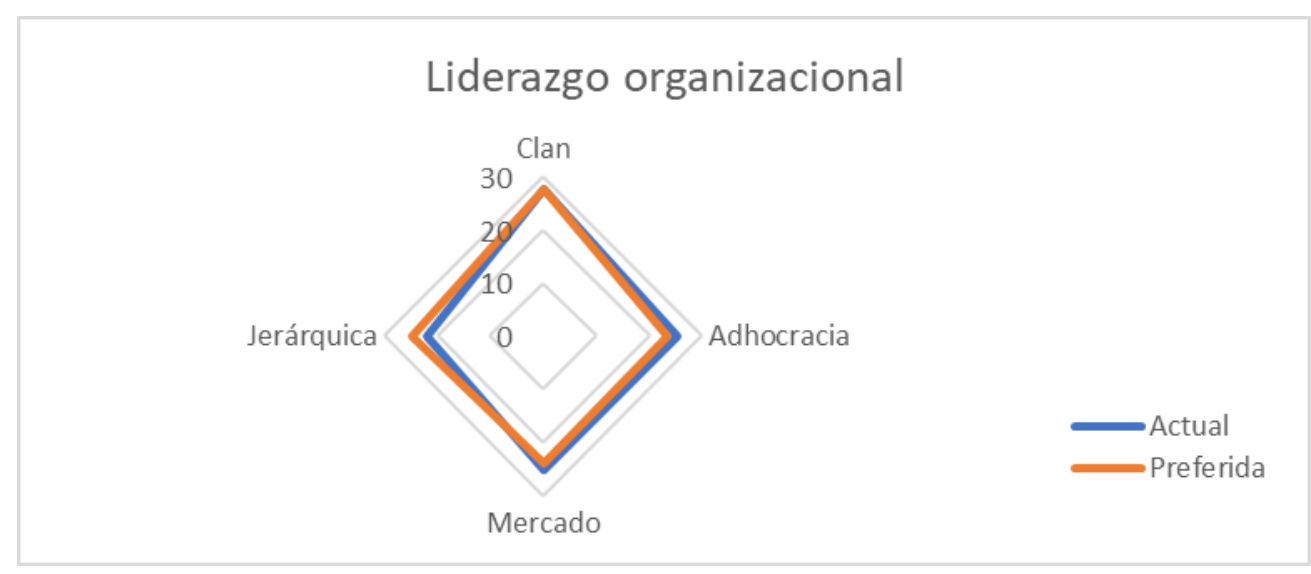

Fuente: Elaboración propia.

En la tabla 3, se muestran los porcentajes correspondientes a los otros tipos de cultura, se puede observar una tendencia hacia una cultura preferida de tipo Jerárquica con un $24.8 \%$.

Tabla 3.

Comparativo por porcentajes, dimensión: Liderazgo Organizacional

\begin{tabular}{lllll}
\hline \multicolumn{1}{c}{ Cultura } & Clan & \multicolumn{2}{c}{ Adhocracia } & \multicolumn{2}{c}{ Mercado } \\
\hline Actual & $\mathbf{2 7 . 6}$ & 25.3 & 25.1 & 21.9 \\
Preferida & $\mathbf{2 7 . 7}$ & 23.4 & 24.1 & $\mathbf{2 4 . 8}$ \\
\hline
\end{tabular}

Fuente: Elaboración propia

El análisis de la dimensión gestión de los empleados, se muestra en la figura 5, donde se puede observar que la gestión se encuentra orientada a una forma de dirigir e influenciar a las personas con el fin de contribuir al logro de las metas organizacionales y de grupo. 
Figura 5.

Comparativo gráfico de la dimensión: Gestión de los Empleados.

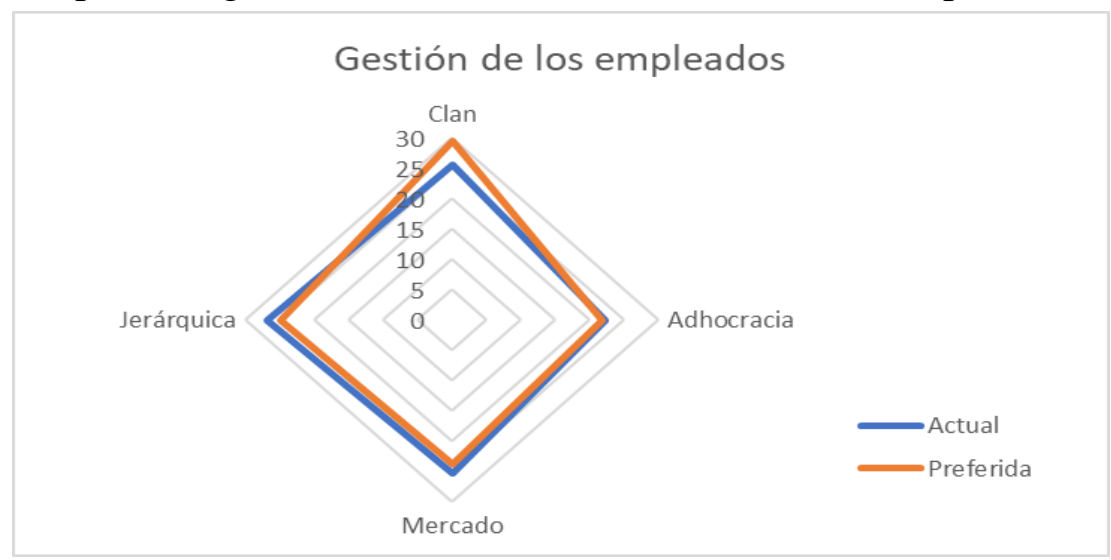

Fuente: Elaboración propia.

En la tabla 4 se puede observar que la dimensión gestión de los empleados obtuvo uno de los porcentajes más altos en lo referente a la cultura preferida con $29.5 \%$, quedando seleccionada la de tipo Clan.

\section{Tabla 4.}

Comparativo por porcentajes, dimensión: Gestión de los Empleados.

\begin{tabular}{|c|c|c|c|c|}
\hline Cultura & Clan & Adhocracia & Mercado & Jerárquica \\
\hline Actual & 25.6 & 22.2 & 25.2 & 27 \\
\hline Preferida & 29.5 & 21.9 & 23.7 & 24.9 \\
\hline
\end{tabular}

Fuente: Elaboración propia

En relación a la cohesión organizacional, esta dimensión engloba la socialización y la unión de los individuos de la organización, estando relacionados con los valores propios del negocio. La figura 6, muestra los resultados del análisis de esta dimensión en la empresa, donde se puede observar que la cultura predomínate es la de Mercado y la preferida es la de Clan.

\section{Figura 6.}

Comparativo gráfico de la dimensión: Cohesión Organizacional.

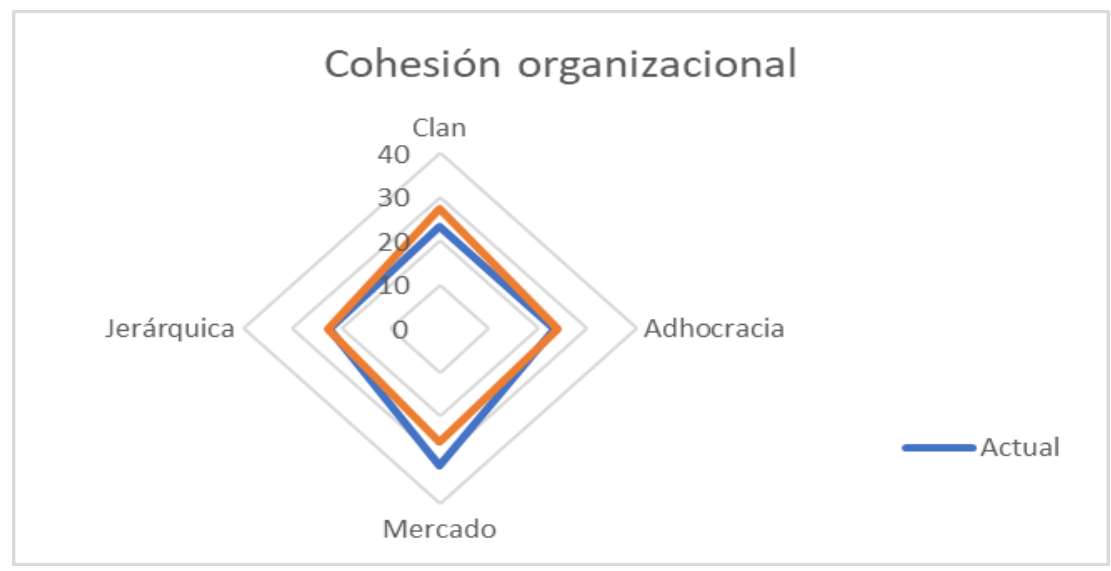


Fuente: Elaboración propia.

La tabla 5, muestra el comparativo de los porcentajes de la dimensión cohesión organizacional, donde se observa un $31.2 \%$ en la cultura actual enfocada a la de Mercado y un 27.4 $\%$ en la cultura preferida enfocada a la de Clan.

\section{Tabla 5.}

Comparativo por porcentajes, dimensión: Cohesión Organizacional.

\begin{tabular}{lrrrr}
\hline \multicolumn{1}{c}{ Cultura } & Clan & \multicolumn{2}{c}{ Adhocracia } & \multicolumn{2}{c}{ Mercado } \\
\hline Actual & 23.3 & 23.2 & $\mathbf{3 1 . 2}$ & 22.3 \\
Preferida & $\mathbf{2 7 . 4}$ & 24.0 & 26.0 & 22.6 \\
\hline
\end{tabular}

Fuente: Elaboración propia.

Por su parte, la dimensión de énfasis estratégico engloba el objetivo en un periodo de tiempo determinado, así como los planes de acción con sus respectivas especificaciones de recursos, tanto humano como técnico para el logro de las metas propuestas. El resultado obtenido del OCAI para esta dimensión, se muestra en la figura 7 , donde se puede observar una preferencia por una cultura de Clan en lugar de una de Mercado.

\section{Figura 7.}

Comparativo gráfico de la dimensión: Énfasis Estratégico.

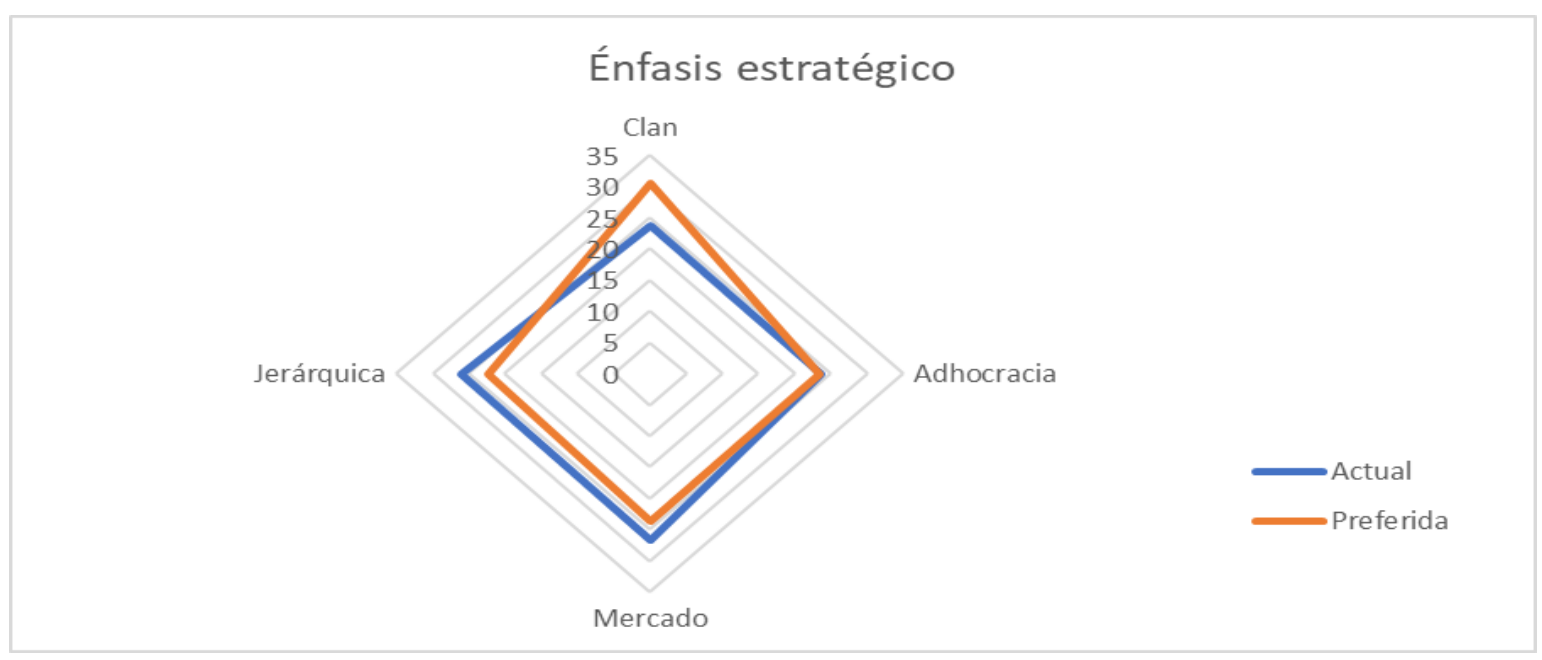

Fuente: Elaboración propia.

La tabla 6, muestra el comparativo de los porcentajes de la dimensión énfasis estratégico, donde se observa un $26.6 \%$ en la cultura actual enfocada a la de Mercado y un $30.5 \%$ en la cultura preferida enfocada a la de Clan.

Tabla 6.

Comparativo por porcentajes, dimensión: Énfasis Estratégico. 


\begin{tabular}{|c|c|c|c|c|c|}
\hline Cultura & Clan & & Adhocracia & Mercado & Jerárquica \\
\hline Actual & & 23.6 & 23.6 & 26.6 & 26.1 \\
\hline Preferida & & 30.5 & 23.4 & 23.7 & 22.4 \\
\hline
\end{tabular}

Fuente: Elaboración propia.

Finalmente, la dimensión de criterios de éxito, presenta una cultura actual dominante enfocada a la de Mercado, sin embargo, en la figura 8, se puede observar con respecto a la cultura deseada una orientación de los empleados a cambiar hacia una cultura de tipo Clan, además que los resultados muestran que también prefieren una Adhocrática.

\section{Figura 8.}

Comparativo gráfico de la dimensión: Criterios de Éxito.

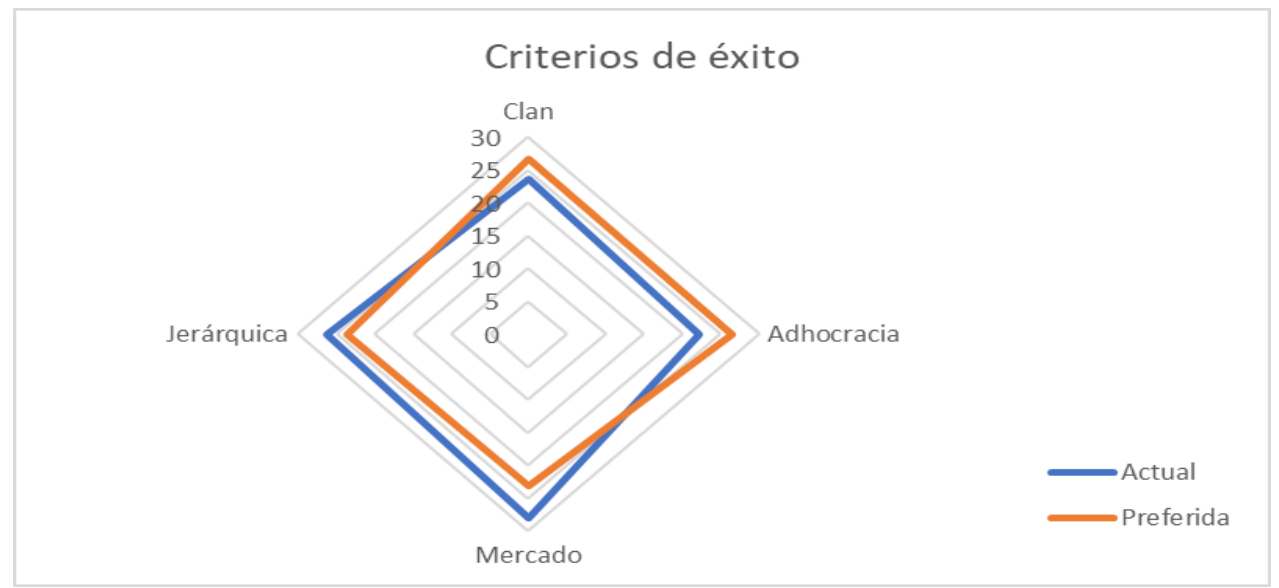

Fuente: Elaboración propia.

En relación a lo anterior, la tabla 7, muestra el comparativo de los porcentajes, donde se observa que la cultura actual predominante para la dimensión criterios de éxito presenta un $28 \%$ enfocándose a la de Mercado y para la cultura preferida se tiene un $26 \%$ tanto para la de Clan como para la Adhocrática.

Tabla 7.

Comparativo por porcentajes, dimensión: Criterios de Éxito.

\begin{tabular}{|c|c|c|c|c|}
\hline Cultura & Clan & Adhocracia & Mercado & Jerárquica \\
\hline Actual & 23.5 & 22.1 & 28 & 26.3 \\
\hline Preferida & 26.6 & 26.6 & 23.1 & 23.7 \\
\hline
\end{tabular}

Fuente: Elaboración propia.

\subsection{Discusión}


Después de la interpretación de los resultados se procede a la discusión de los mismos a partir del objetivo general de la investigación, el cual consiste en diagnosticar la cultura organizacional actual y preferida de la empresa estudiada, además de identificar si existen diferencias entre las dimensiones de la cultura organizacional. En este sentido, Villamarín et al., (2019), manifiesta que conocer la cultura organizacional actual y deseada de una organización, le permitirá al líder de empresa, generar mecanismos de innovación que impulse una cultura de aprendizaje en todos sus equipos de trabajo, de lo contrario, cuando las organizaciones no tienen una orientación hacia la adaptación al cambio, el conocer el modelo de cultura actual y deseada no permite el desarrollo de acciones claves que oriente hacia el aprendizaje organizacional.

En el diagnóstico de la cultura organizacional realizado por Villarreal et al., (2012), en hospitales de la ciudad de Durango, México, manifiesta que la cultura actual predominante fue la de mercado, la cual, coincide con los resultados del presente estudio, así mismo, como cultura deseada manifiesta, que los trabajadores prefieren la de tipo clan, coincidiendo nuevamente con los resultados que arroja esta investigación. Por su parte, las dimensiones analizadas también presentan una alta orientación a la cultura tipo clan como deseada, percibiendo la necesidad de modificar su forma de trabajar y sobre todo cambiar la forma de relacionarse dentro de la organización, con mayor enfoque al desarrollo personal y un trabajo en equipo consolidado, resultados que coinciden con el presente estudio.

Por su parte, en el estudio realizado por Cárdenas (2019) en una institución financiera, manifiesta también que los colaboradores tanto operativos como directivos, de acuerdo a su perspectiva, predomina una cultura de mercado en sus áreas de trabajo, de la misma forma, coincide con los resultados de esta investigación. Así mismo, en relación a las dimensiones de la cultura organizacional no se presentan diferencias significativas en la percepción, encontrando semejanzas con los resultados que presenta Lázaro et al. (2017)

Por otro lado, Cifuentes y Londoño (2017) mencionan en sus estudios que predomina los tipos de cultura clan y adhocrática, siendo esta última aquella que favorece al aprendizaje e innovación la cual, tiene relación con la cultura de aprendizaje. A su vez, Carrasco et al., (2015), sostiene que las organizaciones familiares se orientan generalmente a una cultura clan, mientras que en las empresas no familiares predomina los tipos de cultura mercado y jerárquica. Por su parte, Tortolero et al. (2020) señala que la cultura organizacional tiene un alto grado de asociación con la gestión del conocimiento, por lo tanto, conocer la cultura deseada de acuerdo a la perspectiva de los trabajadores permite que el líder de la organización promueva actividades de gestión del conocimiento en el cumplimiento de sus objetivos a partir de una cultura organizacional alineada a la estrategia de la empresa, un equipo de gerencia con un buen liderazgo y el uso óptimo de las tecnologías de la información. En este sentido, Llanos y Bell (2018), señalan que la cultura organizacional influye en el comportamiento de los miembros de una organización, proporcionando los lineamientos sobre la forma de hacer las cosas y los límites de cumplimiento en función de su responsabilidad.

\section{CONCLUSIONES}

El empleo del OCAI en la presente investigación, fue de gran utilidad para diagnosticar la cultura organizacional de la empresa estudiada. Este cuestionario permite concluir que la cultura dominante fue la de mercado y la cultura deseada fue la de clan.

Los resultados ponen de manifiesto un desfase cultural, es decir, la cultura actual es distinta a la cultura deseada, dejando ver la presencia de áreas de oportunidad, las cuales, pueden ser mejoradas o subsanadas existiendo voluntad por parte de la gerencia y los trabajadores.

En relación a la dimensión liderazgo organizacional, se concluye que la cultura actual manifiesta un líder de puertas abiertas, motivador, dejando ver el deseo del grupo para mantenerlo 
unido. Sin embargo, también existe una tendencia a la necesidad de un líder con mayor capacidad para dar instrucciones claras y precisas, con la suficiente habilidad y autoridad para resolver problemas cotidianos, cuyas decisiones puedan ser independientes y rápidas.

Por su parte, la dimensión de gestión de los empleados, al presentar como resultado una tendencia de cultura preferida tipo clan, se concluye que existe la necesidad de mejorar la comunicación en todas las direcciones, permitiendo una mayor participación e involucramiento del personal en la resolución de problemas y la toma de decisiones, pero sobre todo la necesidad de generar equipos de trabajo sólidos.

En el caso de la dimensión cohesión organizacional, se concluye que existe la necesidad de desarrollar habilidades y valores con respeto, empatía, solidaridad, compromiso y comunicación asertiva, no solo con los compañeros inmediatos, sino con otras áreas de la empresa, incluyendo un involucramiento mayor de la gerencia y buscando fortalecer el pegamiento social de la empresa y de esta manera alcanzar los objetivos organizacionales. A su vez, la dimensión énfasis estratégico, al tener una preferencia de cultura de tipología clan, se hace manifiesto la importancia que tiene la confianza hacia el trabajador, ya que existe la necesidad de apertura hacía el individuo para empoderarlo y llevarlo a tener una mayor participación e involucramiento en el logro de las metas organizacionales.

Finalmente, la dimensión de criterios de éxito, al presentar una tendencia de cultura preferida a dos de las tipologías, que son la clan y la adhocrática, muestra el deseo de contar con el desarrollo del recurso humano a través de una constante capacitación que permita generar equipos de trabajo eficientes para mejorar la calidad de los productos y el servicio al cliente.

El desarrollo del presente estudio, el cual aborda la temática cultural en el contexto local, permitió realizar un contraste teórico y comprobar la conveniencia del uso del instrumento para el contexto latinoamericano y sobre todo mexicano, el cual es propuesto dentro de la literatura especializada. Dentro de las limitaciones de la investigación, se encuentra que solo se contempla una organización, por lo que es necesario aumentar la cantidad de empresas diagnosticadas y sobre todo con un mayor número de trabajadores, con el objetivo de tener más datos que permitan generalizar los resultados a un contexto más amplio para identificar sus diferencias o semejanzas entre las organizaciones estudiadas. Así mismo, se debe tomar en cuenta otras variables que pudieran influenciar en la gestión del conocimiento y el aprendizaje organizacional para que los líderes organizacionales cuenten con mayor información para elaborar planes de acción estratégicos que impulsen los procesos de innovación y la adaptación al cambio.

Este análisis, se encuentra exento del conocimiento de la existencia de micro culturas o subculturas dentro de cualquier organización, por lo cual, la presente investigación permitirá que surjan futuros estudios con la profundización del tema cultural en las organizaciones mexicanas, pero desde una perspectiva ad hoc, la cual, considere el análisis de modo más objetivo y riguroso.

\section{REFERENCIAS}

Albarrán-Carrera, D. (2010). Cultura Organizacional y Representación Social del Discapacitado. Caso centro de rehabilitación DIF-Mazatlán. (Tesis doctoral). Universidad Autónoma Metropolitana, México D.F., México.

Andrade, H. (2011). Cambio o Fuera: Dirigir en el siglo XXI. Palibrio.

Guizar, R. (2004). Desarrollo organizacional. México, DF: Editorial McGraw Hill, 3.

Cárdenas, V. (2019). Percepción de la cultura organizacional según Cameron y Quinn en la Tienda 512 de un Banco en el distrito de El Tambo, Huancayo. Tesis para optar el título de Licenciado en Administración, Escuela Académico Profesional de Administración, Universidad Continental, Huancayo, Perú.

Carrasco, A.J., Sánchez, G., Danvila, I., \& Sastre, M.Á. (2015). Orientaciones competitivas culturales en la empresa familiar/Competitive cultural orientation in the family business. Cuadernos de Estudios Empresariales, $25,49$. 
Cameron, K. S., \& Quinn, R. E. (2011). Diagnosing and changing organizational culture: Based on the competing values framework. John Wiley \& Sons.

Chuc, F. (2017). Un modelo adecuado para diagnosticar la Cultura Organizacional. IC Investigación, 12, 117-135.

Chuc, F. (2018). Aplicación de un modelo para determinar la Cultura Organizacional en un sistema educativo. Investigación Acción(14), 45-59.

Cifuentes, L., \& Londoño, M. (2017). Rasgos Identificativos que promueven la Cultura de la Innovación en las. Revista Interamericana de psicología ocupacional, 33-48. doi:10.21772/ripo.v36n1a03

Florido, J. S. V., \& Zesaty, J. (2010). Los tipos de cultura organizacional y el rendimiento de las pymes en Aguascalientes. In congreso.investiga.fca.unam.mx.

Gómez-Romero, J. G. I. (2008). La generación de conocimiento organizacional en la micro, pequeña y mediana empresas (MIPYMES) de Durango. México Durango: Editorial UJED.

González, Y., Liquidano, M., \& Carlos-Ornelas, C. (2012). Cultura organizacional actual y preferida y estilo de liderazgo en una empresa gasera de Aguascalientes. Retos de las Ciencias Administrativas desde las Economías Emergentes: Evolución de Sociedades. Monterrey: ITESM.

Hernández, R., \& Valencia, S. M. (2012). Exploración factorial del clima y la cultura organizacional em el marco del modelo de los valores en competencia. PsiqueMag, 1(1), 11-47.

Hodge, B. J., Anthony, W. P., \& Gales, L. M. (1998). Teoría de la organización: Un enfoque estratégico. Pearson Prentice Hall.

Ibarra Michel, J. P. (2017). Cultura organizacional orientada a la sustentabilidad en empresas hoteleras de Mazatlán. (Tesis doctoral). Universidad de Occidente, Culiacán, Sinaloa.

Ibarra-Michel, J. P., Velarde-Valdez, M., Olmos-Martínez, E., \& Santillan-Nuñez, M. A. (2019). Evaluación de la cultura organizacional en empresas hoteleras sostenibles de Mazatlán.

Lázaro, L., Romero, R., Saavedra, J., \& Vásquez, M. (2017). La cultura organizacional en tres bancos comerciales del departamento de Lambayeque. Obtenido de Pontificia Universidad Católica del Perú.[Tesis para obtener el grado de magíster en administración estratégica de empresas]: http://tesis.pucp.edu. pe/repositorio/bitstream/handle/20.500, 12404, 8764.

Llanos, M. del P., y Bell, R. E. (2018). La cultura organizacional: Abordaje conceptual y delimitación de variables para su estudio. Revista Científica ECOCIENCIA, 5(2), 1-19.

Minsal-Pérez, D., \& Pérez-Rodríguez, Y. (2007). Hacia una nueva cultura organizacional: la cultura del conocimiento. Acimed, 16(3), 0-0.

Ouchi, W. (1992). Teoría Z: como pueden las empresas hacer frente al desafío japones. Theory Z: how american business can meet the japanese challenge.

Pacheco Espejel, A. A. (2015). Intervención organizacional, primeras aproximaciones conceptuales.

Pettigrew, A. M. (1979). On studying organizational cultures. Administrative science quarterly, 24(4), 570-581.

Salas-Arbeláez, L., Solarte, M. G., \& Vargas, G. M. (2017). Efecto de la cultura organizacional en el rendimiento de las PYMES de Cali. Suma de negocios, 8(18), 88-95.

Schein, E. H. (2010). Organizational culture and leadership (Vol. 2). John Wiley \& Sons.

Tarapuez-Chamorro, E. (2016). Las dimensiones culturales de Geert Hofstede y la intención emprendedora en estudiantes universitarios del departamento del Quindío (Colombia). Pensamiento \& Gestión, (41), 60-90.

Tortolero, R., Figueroa, E.G., \& Villarreal, F.M. (2020). Modelo de regresión lineal múltiple de la gestión del conocimiento, con la cultura organizacional, el liderazgo y las tecnologías de la información y la comunicación, en trabajadores de una empresa de la Cd. de Durango, Durango, México. Hitos de Ciencias Económico Administrativas, 26(76), 266-284.

Villamarin, M. F., Tejera, E., \& Ramos, V. (2019). La cultura organizacional actual y deseada y su relación con la cultura de aprendizaje: aplicación del modelo de valores competitivos de Quinn. La cultura, 40(42).

Villarreal, M. D., Villareal, F. M., \& Briones, E. E. (2012). Diagnóstico de la Cultura Organizacional en un Hospital de Zona en Durango. Conciencia Tecnológica, (44), 23-29. 